\title{
BMJ Open Use of health services in the last year of life and cause of death in people with intellectual disability: a retrospective matched cohort study
}

\author{
Kate Brameld, ${ }^{1,2}$ Katrina Spilsbury, ${ }^{2}$ Lorna Rosenwax, ${ }^{3}$ Helen Leonard, ${ }^{4}$ \\ James Semmens ${ }^{2,5}$
}

To cite: Brameld K, Spilsbury K, Rosenwax L, et al. Use of health services in the last year of life and cause of death in people with intellectual disability: a retrospective matched cohort study. BMJ Open 2018;8:e020268. doi:10.1136/ bmjopen-2017-020268

- Prepublication history for this paper is available online. To view these files, please visit the journal online (http://dx.doi. org/10.1136/bmjopen-2017020268).

Received 26 0ctober 2017 Revised 12 December 2017 Accepted 22 January 2018

\section{Check for updates}

${ }^{1}$ Curtin-Monash Accident Research Centre, Faculty of Health Sciences, Curtin University, Bentley, Western Australia, Australia

${ }^{2}$ Centre for Population Health Research, Faculty of Health Sciences, Curtin University, Perth, Western Australia, Australia

${ }^{3}$ School of Occupational Therapy and Social Work, Faculty of Health Sciences, Curtin University, Perth, Western Australia, Australia

${ }^{4}$ Telethon Kids Institute, Subiaco, Western Australia, Australia

${ }^{5}$ The Institute for Health

Research, The University of Notre Dame, Fremantle, Western Australia, Australia

Correspondence to

Dr Kate Brameld;

kate.brameld@curtin.edu.au

\section{ABSTRACT}

Objective To describe the cause of death together with emergency department presentations and hospital admissions in the last year of life of people with intellectual disability.

Method A retrospective matched cohort study using de-identified linked data of people aged 20 years or over, with and without intellectual disability who died during 2009 to 2013 in Western Australia. Emergency department presentations and hospital admissions in the last year of life of people with intellectual disability are described along with cause of death.

Results Of the 63508 deaths in Western Australia from 2009 to 2013, there were 591 (0.93\%) decedents with a history of intellectual disability. Decedents with intellectual disability tended to be younger, lived in areas of more social disadvantage, did not have a partner and were Australian born compared with all other decedents. A matched comparison cohort of decedents without intellectual disability $(n=29713)$ was identified from the general population to improve covariate balance. Decedents with intellectual disability attended emergency departments more frequently than the matched cohort (mean visits 3.2 vs 2.5) and on average were admitted to hospital less frequently (mean admissions 4.1 vs 6.1), but once admitted stayed longer (average length of stay 5.2 days vs 4.3 days). People with intellectual disability had increased odds of presentation, admission or death from conditions that have been defined as ambulatory care sensitive and are potentially preventable. These included vaccine-preventable respiratory disease, asthma, cellulitis and convulsions and epilepsy. Conclusion People with intellectual disability were more likely to experience potentially preventable conditions at the end of their lives. This indicates a need for further improvements in access, quality and coordination of healthcare to provide optimal health for this group.

\section{INTRODUCTION}

A review of the research literature into the health of people with intellectual disability in comparison to the general population highlighted a cascade of disparities. ${ }^{1}$ The disparities are to some extent expected due to the

\section{Strengths and limitations of this study}

- We provide a population perspective on use of emergency and hospital services for people with intellectual disability in their last year of life

- Health service use and causes of death are compared with a matched population cohort.

- A potential limitation of our study is our ability to accurately identify people with intellectual disability from a disease register and administrative health records.

- We have not accounted for the severity of intellectual disability.

serious and complex comorbid conditions that can co-occur with intellectual disability. However, many problems with access to healthcare have been documented, such as a lack of uptake of health-promoting activities and preventative care, ${ }^{2}$ inadequate healthcare in the community ${ }^{3}$ and problems with access to hospital care. ${ }^{3-5}$ These can in turn lead to exacerbation of conditions, particularly those that are ambulatory care sensitive, for which the institution of effective management and treatment could prevent the need for emergency presentation or hospital admission ${ }^{6}$ and contribute to a reduction in premature mortality in this population. ${ }^{7-10}$

The health inequities and disadvantage in a population with intellectual disability were highlighted by the British charity Mencap in their March 2007 report 'Death by Indifference'. ${ }^{11}$ The report ascribed the deaths of six people with learning disabilities as a consequence of institutional discrimination within the National Health Service, leading to a confidential enquiry into premature deaths of people with intellectual disability. ${ }^{8}$ The enquiry determined that of 247 deaths in people with intellectual disability in southwest England between 2010 and 2012, 37\% of the 
deaths were from causes amenable to good-quality healthcare as opposed to $13 \%$ of deaths in the general population of England and Wales. Eighteen recommendations regarding aspects of healthcare for people with intellectual disability were made as a result of the enquiry. ${ }^{8}$ Despite the evidence, it has only been in more recent years that these issues have begun to be addressed in national and international policy statements. ${ }^{12-14}$ Ongoing monitoring is required to ensure that policy is being translated into action and that effective change is occurring.

There is currently limited information on the rates of health service use in people with intellectual disability and none that we could find regarding health service use during the last year of life. A number of studies examined mortality in this population ${ }^{9101516}$ and highlighted a younger age at death, higher mortality rates and potentially avoidable deaths.

The aim of this study was to describe cause of death and place of death together with emergency department (ED) presentations and hospital admissions in the last year of life of people with intellectual disability. We hypothesised that people with intellectual disability would demonstrate different patterns of access to health services in their last year of life compared with people without intellectual disability. The relative odds of presenting to ED, being admitted to hospital and causes of death within diagnostic categories were compared between the cohort of decedents with intellectual disability and a matched comparison cohort, with a specific focus on ambulatory care sensitive conditions. Such information provides baseline data to ensure we improve the health of people with intellectual disability.

\section{METHODS}

This study was a retrospective matched cohort design that used de-identified emergency presentations, hospital admissions from the last year of life and death data linked at the Data Linkage Branch, Western Australian Department of Health, with data from the Intellectual Disability Exploring Answers (IDEA) Database. ${ }^{17}$ The IDEA Database is a population-based data source of information on people with an intellectual disability accessing services from the Disability Services Commission and/or educational support from the Department of Education. Case ascertainment using the IDEA register is thought to be good with the prevalence of intellectual disability in Western Australia measured as 17.0/1000 live births based on data from 1983 to 2005 with follow-up to $2010 .^{18}$ This is consistent with international estimates. ${ }^{19}$ Relying on hospital records alone for case ascertainment in intellectual disability has been demonstrated to have poor sensitivity $(14.9 \%)$ but high specificity $(99.9 \%) \cdot{ }^{20}$ However, by linking these multiple data sources, we believe the identification of intellectual disability is relatively complete in our study, although future work may wish to distinguish between people with and without severe intellectual disability.

\section{Cohort selection}

The study included all people aged 20 years and over with intellectual disability who died in Western Australia during 2009-2013, and a matched comparison cohort of decedents without a history of intellectual disability from the population pool of all decedents also from the period 2009-2013. People with intellectual disability were identified by either having a record on the IDEA database, which includes data from 1953 onwards or if they had a specified International Classification of Diseases (ICD) code $^{21}$ indicating intellectual disability in a hospital morbidity record in any diagnosis field from 1970 to 2008 (ie, excluding the 5-year period before death) or on their death certificate as an underlying or contributing cause of death. The ICD codes used to extract cases were those used by Lin et $a l^{22}$

Comparisons between people who died with intellectual disability and the general population who died without intellectual disability indicated very different demographic profiles. Coarsened exact matching was used to identify a matched comparison cohort of decedents without a history of intellectual disability from the population pool of all decedents. ${ }^{23}$ Matching was performed to create a similar balance in age groups, partner status, sex, indigenous status, quintile of relative disadvantage and country of birth in both the intellectual disability and comparison cohorts. This matching reduced confounding by the matching factors when crude or unadjusted statistical tests were used. After the matching process, there remained 203 matched strata with 591 decedents from the intellectual disability cohort and 29713 decedents from the pool of decedents without intellectual disability became the matched comparison cohort. Observations from the matched comparison cohort were weighted according to the size of the strata and 33204 unmatched decedents without intellectual disability were excluded. Matching and regression models were all adjusted for indigenous status, but we did not have ethical approval to report findings specifically for this population subgroup.

\section{Demographic and health-related variables}

Demographic characteristics from the death certificate included age, gender, marital status, accessibility to services and socioeconomic status. Socioeconomic status was estimated from the Index of Relative Disadvantage (IRSD), which estimates disadvantage of a small geographic area based on 17 different measures including education, income, occupation and unemployment. ${ }^{24}$ Each person's death record is assigned to the small geographic area (Statistical Area Level 1-SLA1 ${ }^{25}$ ) based on a geocode, which is assigned to their record based on their address. Accessibility to services categories were based on the Accessibility/Remoteness Index for Australia, ${ }^{26}$ which measures accessibility to services by taking road distances to nearest service centres and population size into account. These are assigned in the same manner as the IRSD. 
The usual place of residence and the place of death for people with intellectual disability was categorised based on the death certificate data as residential aged care facility, hospital, independent living (including family home), intellectual disability facility or other.

Decedent comorbidity was estimated as the sum of the 31 Elixhauser comorbid conditions recorded in hospital morbidity records over the last 5 years of life. ${ }^{27}$

\section{Diagnostic categories and ambulatory care sensitive conditions}

The main reason for each hospital admission, ED presentation and death was categorised based on the ICD-10-Australian Modification (AM) chapters ${ }^{21}$ and then compared with the matched cohort. As a large proportion of ED presentations outside the metropolitan area in Western Australia are not given an ICD diagnosis code but are classified to a 'major diagnostic category', ${ }^{28}$ the major diagnostic categories were mapped back to ICD chapters where possible. After mapping, $3816(5.1 \%)$ of the ED visits could not be assigned to a diagnostic category and were categorised as unclassified.

Ambulatory care sensitive conditions are those for which effective management and treatment should prevent the need for emergency presentation or hospital admission, and prevent premature mortality. Ambulatory care sensitive conditions were identified from the principal diagnosis variable for hospital admissions and the final diagnosis variable for ED presentations. The ICD-10-AM codes we used to define ambulatory care sensitive conditions were based on those reported by the Australian Institute of Health and Welfare, ${ }^{29}$ Purdy $e t a l^{30}$ and Glover and Ayub. ${ }^{15}$

\section{Statistical methods}

Chi-squared tests of the equality of proportions were conducted to see if there were significant differences in the demographic characteristics of people with and without intellectual disability. A negative binomial distribution was assumed when estimating the mean number of ED presentations, hospital admissions and total length of stay over the last year of life. Multivariate logistic regression models were used to compare the odds of emergency presentation, hospital admission and death for each ICD diagnostic condition between the two cohorts. The models were adjusted for demographic factors including age group, gender, marital status, indigenous status, country of birth, socioeconomic status, accessibility and comorbidity. ${ }^{27}$ As the logistic regression analysis involved multiple comparisons, the Bonferroni correction was applied to identify significant ORs. Thus, $\mathrm{P}$ values were required to be less than 0.003 to be significant at the $95 \%$ level. Strata weights were applied in all estimation procedures involving the matched comparison cohort. Variables used in the matching process were also included in regression analyses for full adjustment because the matching process used coarsened versions of these variables. Specific medical conditions within ICD chapters where people with intellectual disability had an increased odds of presentation, admission or death were also examined.

For analyses of the ambulatory care sensitive conditions, the number of ED presentations or hospital admissions for each decedent for each of the 19 different conditions was summed over the last year of life. Estimation of the adjusted relative rates of ED presentations and hospital admissions in the last year of life for each ambulatory care sensitive conditions was performed by modelling the mean number of events per decedent assuming a negative binomial distribution. Logistic regression was used to model the odds of an ambulatory care sensitive care condition recorded as the underlying cause of death. All models were adjusted for matching variables and the number of comorbid conditions.

Data management and statistical analyses were conducted with SAS V.9.4 for Windows (SAS Institute, Cary, North Carolina, USA) and Stata Statistical Software: Release 14 (Stata Corp, College Station, Texas, USA). Ethics permission was granted by the Department of Health, Western Australia (2015/35) and Curtin University Human Research Ethics Committee (4881).

\section{RESULTS}

There were $591(0.93 \%)$ people with intellectual disability who died aged 20 years or over between 2009 and 2013 identified from the population pool of 63508 decedents. The majority $(n=343,58 \%)$ of decedents with intellectual disability were living in independent living accommodation at the time of death with a further $32 \% \quad(n=189)$ living in residential aged care facilities (RACFs), and $10 \%(\mathrm{n}=59)$ in other types of accommodation including hospital and intellectual disability care facilities. Just under half (47\%) died in hospital, 22\% died in independent living accommodation, 23\% in an RACF and $8 \%$ in other.

The sociodemographic profile of the intellectual disability cohort varied significantly from the general population of decedents (table 1). Decedents with intellectual disability tended to be younger, live in areas of more social disadvantage, not have a partner and be Australian born compared with the population comparison group. There was no difference between decedents with and without intellectual disability in terms of accessibility to services or sex distribution.

To minimise the effect of the different sociodemographic structure of decedents with and without intellectual disability, a matched comparison cohort of 29713 decedents without intellectual disability were identified from the decedent pool. After matching, the cohort of decedents with intellectual disability and the matched comparison cohort had a similar age, sex, level of disadvantage, partner status, indigenous status and country of birth status structure. This allowed comparison of summary characteristics of ED presentations and hospital admissions between the two cohorts with 
Table 1 Demographic and matching variables for the intellectual disability cohort and the two comparison cohorts

\begin{tabular}{|c|c|c|c|c|c|}
\hline & \multicolumn{2}{|c|}{$\begin{array}{l}\text { Population } \\
\text { comparison } \\
\text { cohort } \\
\text { ( } \mathrm{n}=62917 \text { ) }\end{array}$} & \multicolumn{2}{|c|}{$\begin{array}{l}\text { Intellectual } \\
\text { disability } \\
\text { cohort } \\
(n=591)\end{array}$} & \multirow[b]{2}{*}{$P$ value } \\
\hline & $\mathbf{N}$ & $\%$ & $\mathbf{N}$ & $\%$ & \\
\hline \multicolumn{6}{|c|}{ Age at death (years) } \\
\hline 20-29 & 968 & 1.5 & 55 & 9.3 & $<0.001$ \\
\hline $30-39$ & 1385 & 2.2 & 55 & 9.3 & \\
\hline $40-49$ & 2550 & 4.1 & 88 & 14.9 & \\
\hline $50-59$ & 4752 & 7.6 & 146 & 24.7 & \\
\hline $60-69$ & 7887 & 12.5 & 115 & 19.5 & \\
\hline $70-79$ & 12699 & 20.2 & 79 & 13.4 & \\
\hline $80-89$ & 21120 & 33.6 & 45 & 7.6 & \\
\hline $90+$ & 11556 & 18.4 & 8 & 1.4 & \\
\hline \multicolumn{6}{|l|}{ Sex } \\
\hline Male & 33089 & 52.6 & 332 & 56.2 & 0.082 \\
\hline Female & 29828 & 47.4 & 259 & 43.8 & \\
\hline \multicolumn{6}{|c|}{ Index of Relative Social Disadvantage } \\
\hline $\begin{array}{l}\text { Most } \\
\text { disadvantaged }\end{array}$ & 16604 & 26.4 & 208 & 35.2 & $<0.001$ \\
\hline $\begin{array}{l}\text { More } \\
\text { disadvantaged }\end{array}$ & 13344 & 21.2 & 140 & 23.7 & \\
\hline Average & 10926 & 17.4 & 98 & 16.6 & \\
\hline $\begin{array}{l}\text { Less } \\
\text { disadvantaged }\end{array}$ & 9663 & 15.4 & 64 & 10.8 & \\
\hline $\begin{array}{l}\text { Least } \\
\text { disadvantaged }\end{array}$ & 8404 & 13.4 & 49 & 8.3 & \\
\hline Unknown & 3976 & 6.3 & 32 & 5.4 & \\
\hline \multicolumn{6}{|l|}{ Partner status } \\
\hline Not partnered & 30958 & 49.2 & 520 & 88.0 & $<0.001$ \\
\hline Partnered & 31959 & 50.8 & 71 & 12.0 & \\
\hline \multicolumn{6}{|l|}{ Accessibility index } \\
\hline Major cities & 45904 & 73.0 & 445 & 75.3 & 0.245 \\
\hline Inner regional & 5316 & 8.4 & 39 & 6.6 & \\
\hline Outer regional & 5200 & 8.3 & 45 & 7.6 & \\
\hline Remote & 1744 & 2.8 & 23 & 3.9 & \\
\hline Very remote & 828 & 1.3 & 8 & 1.4 & \\
\hline Unknown & 3925 & 6.2 & 31 & 5.2 & \\
\hline \multicolumn{6}{|l|}{ Australian born } \\
\hline No & 26535 & 42.2 & 82 & 13.9 & $<0.001$ \\
\hline Yes & 36382 & 57.8 & 509 & 86.1 & \\
\hline
\end{tabular}

reduced confounding effects (table 2). Compared with decedents in the matched cohort, decedents with intellectual disability tended to attend ED more frequently (3.2 vs 2.5) but slightly fewer of their ED visits led to admission to hospital ( $55 \%$ vs $58 \%$ ). The ED visits by people with intellectual disability were less often triaged as resuscitation or non-urgent and they presented more often with respiratory, neurological and drug-related and alcohol-related symptoms than the matched cohort (table 2).

In the last year of life, decedents with intellectual disability on average were admitted to hospital less frequently than the matched comparison cohort, but once admitted they stayed longer (table 2). Of the 2433 hospital admissions by decedents with intellectual disability in the last year of life, almost half were classified as emergency admissions, whereas of the 180141 hospital admissions by the matched cohort, only one-third were classified as emergency admissions. Decedents with intellectual disability were less likely to receive hospital-based specialist palliative care but more likely to have hospital stays involving intensive care and ventilator support.

The most common presenting symptoms for ED presentations for both the ID and matched cohorts were pain and respiratory symptoms (table 2). The most common final diagnoses made for ED presentations (table 3) for people with intellectual disability were respiratory conditions, symptoms, signs and abnormal clinical findings and then factors influencing health status and contact with health services. This latter category included people who went to the ED and were triaged but then left without being treated $(29 \%)$, those attending for medical observation and evaluation (26\%) and follow-up examinations $(11 \%)$. In contrast, in the matched cohort, the most common final diagnoses were circulatory and respiratory conditions, symptoms and signs, and injury and poisoning.

The most frequent ICD chapter coded in hospital admissions for both the ID and matched cohorts was 'factors influencing health status' with multiple admissions for dialysis and chemotherapy by the same subsets of patients, $20 \%(n=5,975)$ of the matched cohort and $10 \%(\mathrm{n}=57)$ of the ID cohort, accounting for the majority of these (table 3). Hospital admissions for respiratory conditions were more frequent in the intellectual disability cohort, whereas hospital admission for cancers were more frequent in the matched cohort.

The most frequently recorded causes of death for people with intellectual disability were circulatory disorders $(19.6 \%)$, cancer $(17.4 \%)$ and nervous disorders $(13.9 \%)$ (table 3$)$. This compares with cancers $(31.2 \%)$, external causes of death $(22.9 \%)$ and circulatory conditions $(20.8 \%)$ in the matched cohort.

After adjusting for sociodemographic factors and comorbidity, people with an intellectual disability had increased odds of presentation at ED, hospital admission and death from diseases of the respiratory and nervous systems (table 4). They had increased odds of ED presentation and hospital admission from infectious and parasitic diseases and increased odds of ED presentation alone for endocrine and genitourinary conditions. In contrast, they had a decreased odds of hospital admission and death from cancer and external causes but much increased odds of death from congenital abnormalities 
Table 2 Characteristics of ED presentations and hospital admissions in the last year of life for the intellectual disability and matched comparison cohorts

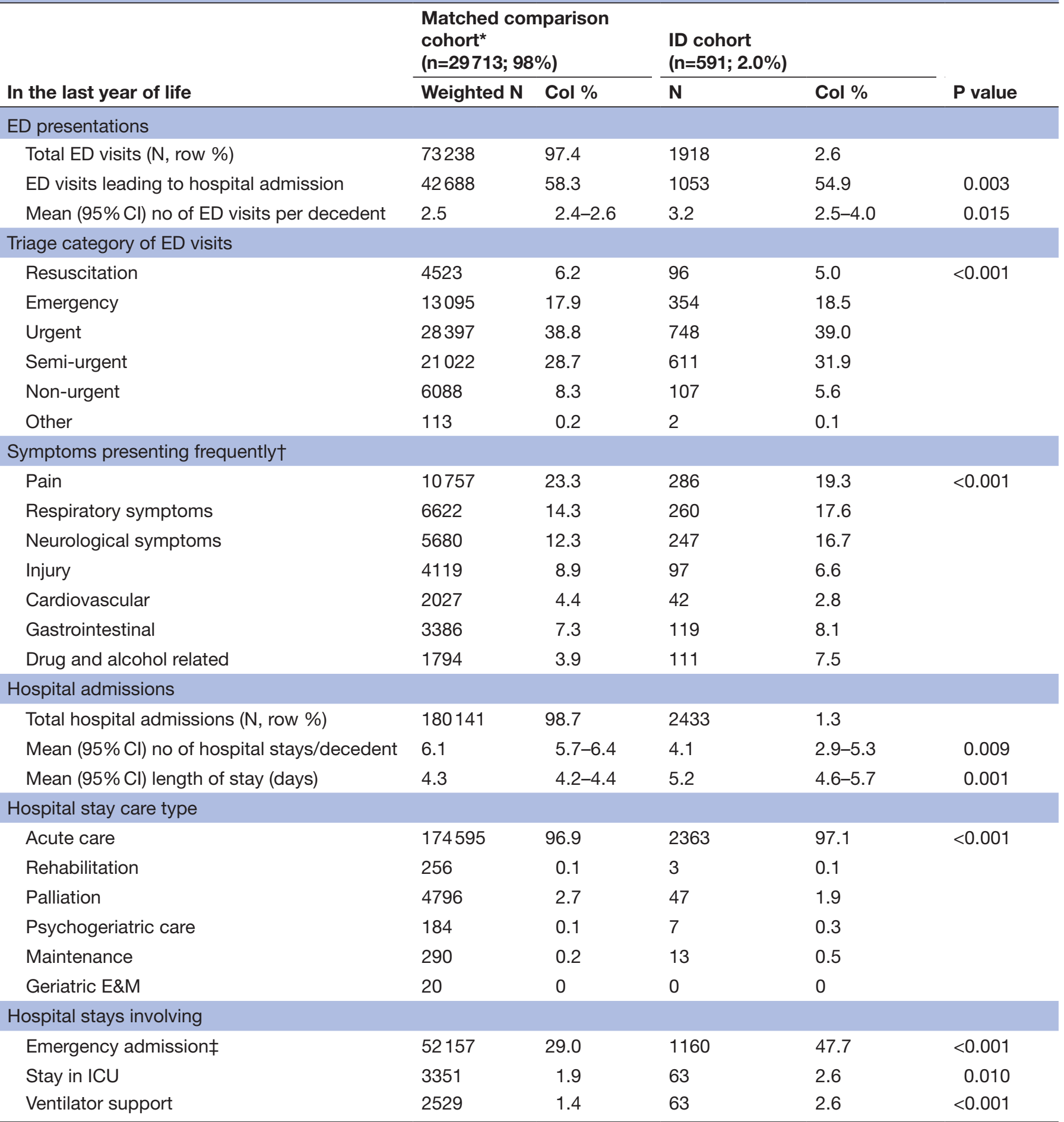

*Matched strata weights applied; therefore, values for $\mathrm{N}$ are those estimated as if the comparison cohort had the same age, sex, socioeconomic, partner, indigenous and country of birth structure as the intellectual disability.

†As a percentage of the $63 \%$ (matched cohort) and $77 \%$ (ID cohort) of ED presentations coded with a presenting symptom.

fIncludes admissions via the ED and direct admissions to specialty care areas such as intensive care and burns units or via the ED of another hospital.

ED, emergency department; E\&M, evaluation and management; ICU, intensive care unit; ID, intellectual disability. 


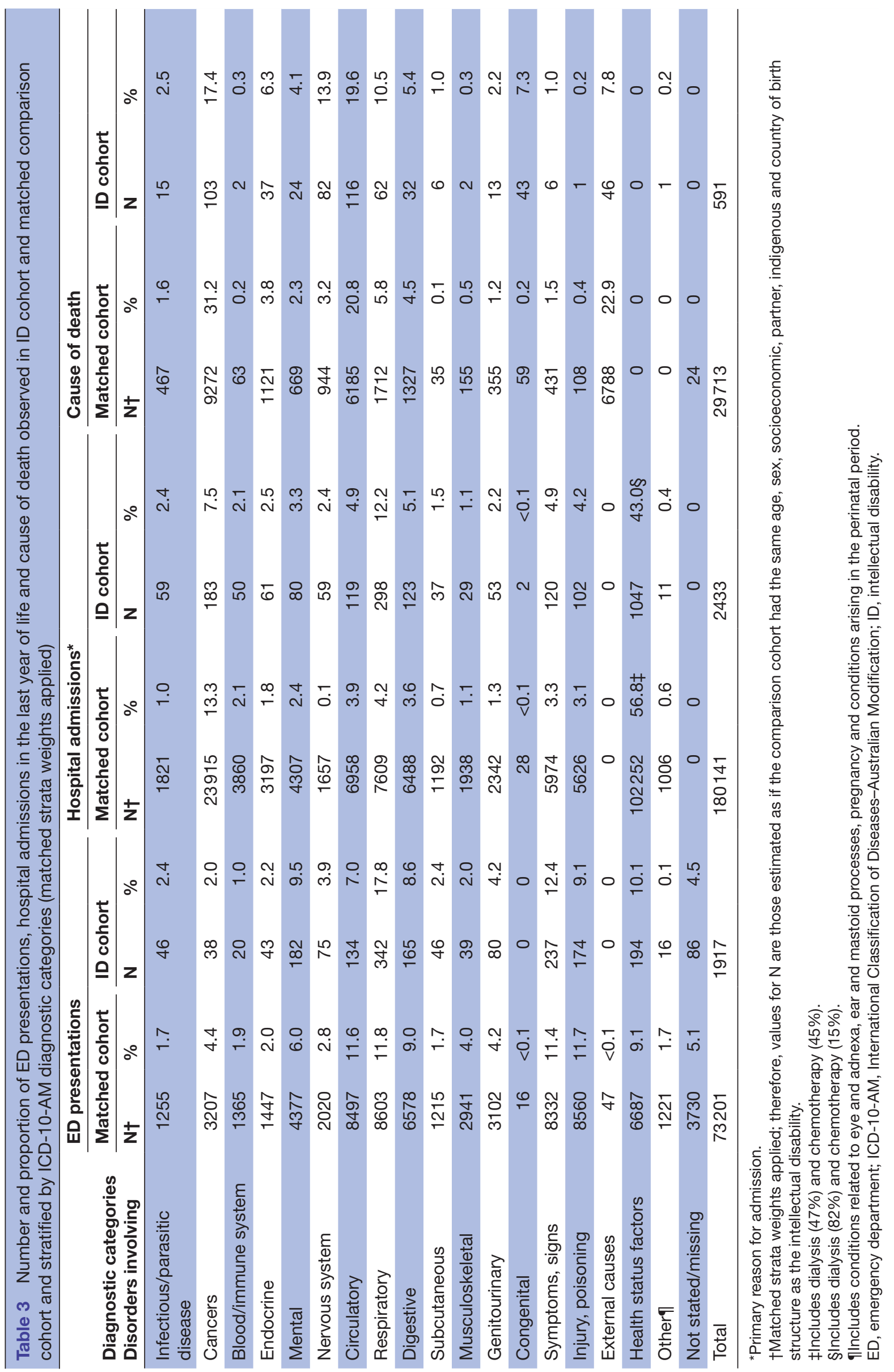




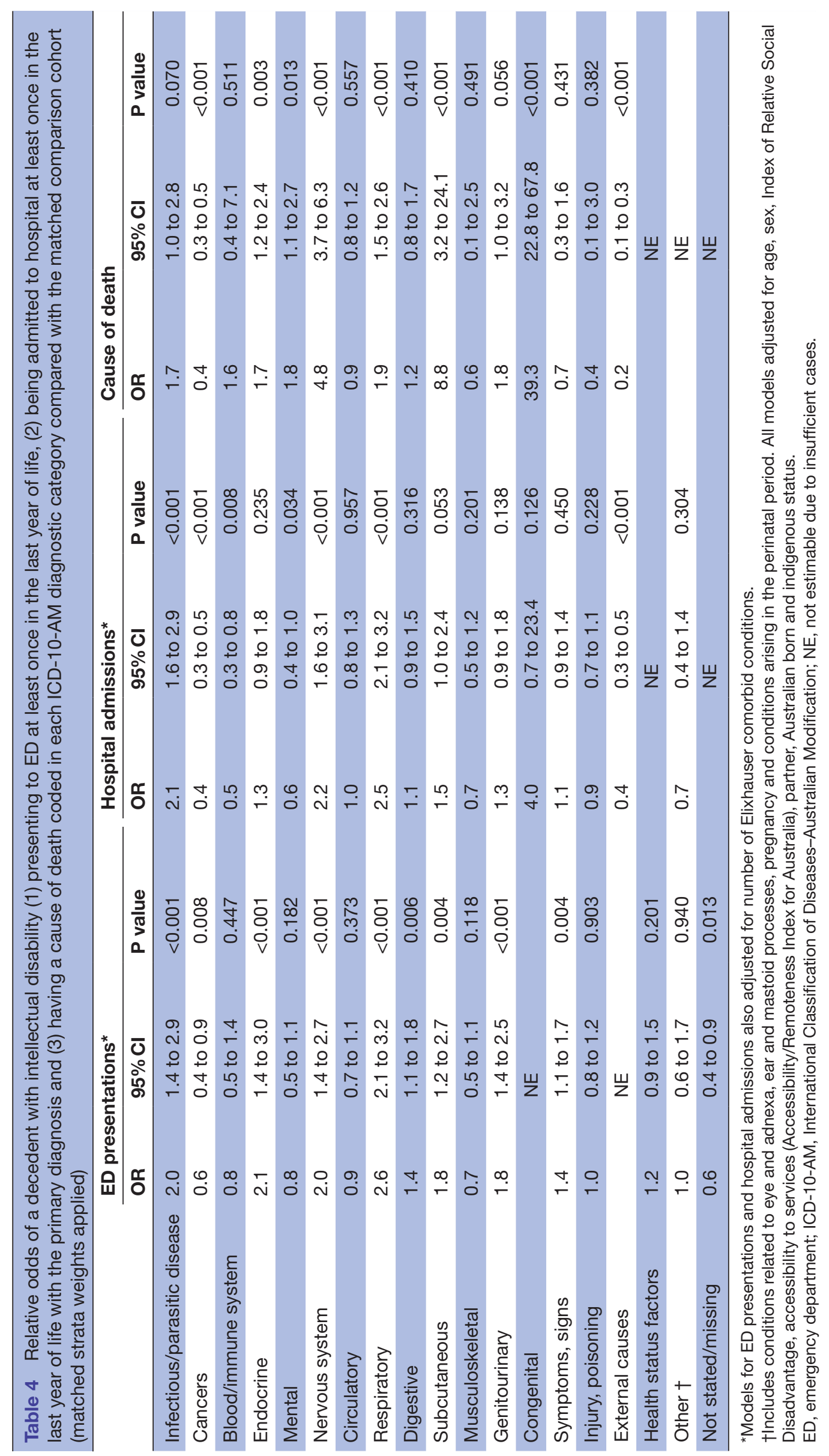


Table 5 Relative rate of ED presentations and hospital admissions for ambulatory care sensitive conditions over the last year of life for decedents with intellectual disability compared with the matched comparison cohort

\begin{tabular}{|c|c|c|c|c|c|c|}
\hline \multirow[b]{2}{*}{ Ambulatory care sensitive conditions } & \multicolumn{3}{|c|}{ ED presentations } & \multicolumn{3}{|c|}{ Hospital admissions } \\
\hline & $\mathbf{R R}$ & $95 \% \mathrm{Cl}$ & $\mathbf{P}$ & $\mathbf{R R}$ & $95 \% \mathrm{Cl}$ & $\mathbf{P}$ \\
\hline \multicolumn{7}{|l|}{ Vaccine preventable } \\
\hline Influenza and pneumonia & 2.6 & 2.0 to 3.4 & $<0.001$ & 2.3 & 1.0 to 5.3 & 0.044 \\
\hline Other vaccine preventable & & NE & & & NE & \\
\hline \multicolumn{7}{|l|}{ Chronic } \\
\hline Diabetes complications & 1.6 & 0.4 to 5.9 & 0.489 & 0.8 & 0.4 to 1.5 & 0.441 \\
\hline Nutritional deficiencies & & NE & & 21.8 & 1.6 to 292.3 & 0.020 \\
\hline Iron deficiency anaemia & 2.9 & 1.2 to 7.1 & 0.018 & 2.0 & 1.0 to 4.2 & 0.055 \\
\hline Hypertension & & $\mathrm{NE}$ & & & $\mathrm{NE}$ & \\
\hline Congestive heart failure & 1.0 & 0.6 to 1.9 & 0.905 & 1.1 & 0.7 to 1.8 & 0.696 \\
\hline Angina & 1.0 & 0.4 to 2.4 & 0.999 & 1.3 & 0.6 to 2.8 & 0.509 \\
\hline Chronic obstructive pulmonary disease & 0.8 & 0.5 to 1.6 & 0.596 & 1.4 & 0.9 to 2.4 & 0.164 \\
\hline Asthma & 4.7 & 2.1 to 10.4 & $<0.001$ & 4.6 & 1.4 to 15.0 & 0.011 \\
\hline \multicolumn{7}{|l|}{ Acute } \\
\hline Dehydration and gastroenteritis & 2.3 & 1.2 to 4.3 & 0.008 & 0.7 & 0.2 to 2.0 & 0.518 \\
\hline Convulsions and epilepsy & 6.2 & 3.9 to 9.9 & $<0.001$ & 4.3 & 2.6 to 6.9 & $<0.001$ \\
\hline Ear, nose and throat infections & 1.9 & 0.8 to 4.0 & 0.122 & 0.0 & $0.0-$. & 0.972 \\
\hline Dental conditions & 0.9 & 0.2 to 3.9 & 0.932 & 1.3 & 0.5 to 3.4 & 0.655 \\
\hline Cellulitis & 1.7 & 1.0 to 2.7 & 0.038 & 2.5 & 1.5 to 4.1 & 0.001 \\
\hline Gangrene & 1.5 & 0.2 to 11.3 & 0.670 & & NE & \\
\hline Perforated/bleeding ulcer & & NE & & & NE & \\
\hline Pyelonephritis & & NE & & & NE & \\
\hline Pneumonitis due to solids/liquids & 17.9 & 11.3 to 28.3 & $<0.001$ & 17.6 & 11.7 to 26.5 & $<0.001$ \\
\hline
\end{tabular}

ED, emergency department; NE, not estimable due to small numbers of events in the cohort of decedents with intellectual disability; RR, rate ratio estimated from negative binomial count model adjusted for age, sex, index of relative disadvantage, partner status, indigenous status, being Australian born and number of comorbid conditions and strata weights applied.

and skin and subcutaneous conditions although there were very low numbers of these.

Relative rates of ED presentations and hospital admission for ambulatory care sensitive conditions in the last year of life for decedents with intellectual disability compared with the matched comparison cohort are shown in table 5. Decedents with intellectual disability were 17 times more likely to present to ED or be admitted to hospital for pneumonitis compared with the matched comparison cohort. While pneumonitis is not usually included as an ambulatory care sensitive condition, we included it as indicated by Glover and Ayub, who report that they are "to some extent preventable". ${ }^{15}$

Decedents with intellectual disability were also around five times more likely to have ED presentations and hospital admissions for asthma, and convulsions and epilepsy and around twice as likely for cellulitis, iron deficiency anaemia and influenza/pneumonia relative to the matched comparison cohort. Decedents with intellectual disability were twice as likely to present to ED with dehydration and/or gastroenteritis, but the rates of hospital admission for this condition were similar for the two cohorts. Rates of hospital admission for nutritional deficiencies were 20 times higher for people with intellectual disabilities, but there were insufficient ED presentations to compare cohorts. There was no difference in the rates of ED presentations or hospital admissions between the two cohorts for any of the heart-related ambulatory care sensitive conditions, chronic obstructive respiratory disease, ear, nose and throat conditions or dental conditions.

The relative odds of having an ambulatory care sensitive condition listed as the underlying cause of death was investigated. After adjusting for matching variables and comorbidity, decedents with intellectual disability had increased odds of dying of influenza/pneumonia (OR 5.3, 95\% CI 2.4 to 11.8 ), convulsions and epilepsy (OR 9.0, 95\% CI 5.6 to 14.5 ), cellulitis (OR 8.5, 95\% CI 2.5 to 29.4), pneumonitis due to solids or liquids (OR 9.9, 95\% CI 5.1 to 19.3 ) and possibly asthma (OR 2.3, $95 \%$ CI 1.0 to 5.2). No difference in the odds of dying from other ambulatory care sensitive conditions was observed for diabetes, circulatory system conditions, chronic obstructive pulmonary disease or dehydration 
and gastroenteritis. There were insufficient cases to estimate the remaining ambulatory care sensitive conditions.

\section{DISCUSSION}

Our study found that decedents with intellectual disability tended to be younger, live in areas of more social disadvantage, not have a partner and be Australian born compared with the general population of people who died over the same period of time. Decedents with intellectual disability tended to live independently or in residential aged care facilities, but many of their deaths occurred in hospital. After comparing with a matched cohort similar in demographic and socioeconomic structure, decedents with intellectual disability attended ED more frequently but had fewer ED visits that led to admission to hospital although a greater proportion of their hospital admissions were from ED. On average, they were admitted to hospital less frequently than the matched comparison cohort, but once admitted they stayed longer. There were some notable differences between the intellectual disability cohort and the matched cohort in their most common diagnoses and in their risk of ED presentation, hospital admission or death by diagnostic group and by ambulatory care sensitive conditions. Presentations and admissions for cancers, circulatory conditions and injuries were more common in the matched cohort compared with respiratory and nervous disorders, presentations for observation and evaluation, and admissions for dialysis in the intellectual disability cohort. People with intellectual disability were also shown to have an increased odds of presentation, admission or death for nervous and respiratory disorders and a decreased odds of presentation, admission or death from cancer.

We found an increased use of acute health services for ambulatory care sensitive conditions in the intellectual disability cohort in comparison to the matched cohort. People with intellectual disability have difficulty accessing appropriate health services ${ }^{31}{ }^{32}$ for conditions such as these for which effective management and treatment should prevent the need for emergency presentation or hospital admission. They include 'chronic conditions where effective care can prevent flare-ups, acute conditions where early intervention can prevent more serious progression; and preventable conditions, where immunisation and other interventions can prevent illness. ${ }^{, 27}$ The higher prevalence ambulatory care sensitive conditions in people with intellectual disability, as has been noted by other authors, ${ }^{633} 34$ is likely to at least partly reflect levels of access to primary care and preventive interventions, issues in care coordination and prevalence of risk factors in the population as documented in the introduction. ${ }^{3-5}$

However, it would be naive to assume that all presentations to ED or hospital admissions for ambulatory care sensitive conditions are unwarranted, particularly in the case of intellectual disability. Reduced ability for effective communication for people with intellectual disability and a lack of understanding of their health concerns by healthcare providers is likely to result in delayed healthcare-seeking behaviour. Longman et al argue that before ambulatory care sensitive conditions are used as a measure of preventable admissions, we need to have a better understanding of the complexity of causes and drivers from both the patient and clinical perspectives. ${ }^{35}$ Supporting this is a recent study showing that while adults with intellectual disability presented to ED more frequently with lower tract respiratory conditions and urinary tract infections, they had similar patterns of primary care use as the general population before admission, ${ }^{36}$ suggesting factors other than primary care access are involved.

Our findings indicate low rates of intervention for cancers in terms of emergency presentations and hospital admissions in the intellectual disability cohort. Lower rates of admission for chemotherapy are also suggested by the relatively lower proportion of hospital admissions for non-emergency reasons in people with intellectual disability. This is despite evidence that there is a similar incidence of cancer in people with and without intellectual disability. ${ }^{37} 38$ This suggests a lower treatment rate once diagnosed for people who have intellectual disability and cancer, consistent with previous research by TuffreyWijne et al and Satgé et al. ${ }^{39} 40$ Tuffrey-Wijne et al reported that some patients with intellectual disability were less likely to be given aggressive treatment because they might have difficulty coping with the treatment regime and it was thus considered not to be in their best interest. Satge $e t a l^{39}$ state that treating lung cancer in patients with intellectual disability is more difficult, partly because late presentation means that more aggressive treatment is required and because issues with communication lead to difficulties in gaining patient understanding and cooperation with treatment. Intellectual disability and related comorbid conditions can also contraindicate one or more cancer treatments such as radiotherapy, chemotherapy, anaesthesia and surgery. ${ }^{39}$

The recent confidential inquiry into premature deaths of people with intellectual disabilities in England found that 'avoidable deaths from causes amenable to change with good quality healthcare are more common in people with intellectual disabilities than in the general population'. ${ }^{8}$ As many of the ambulatory care sensitive conditions responsible for emergency presentations and hospital admissions also appear as the underlying cause of death, for example, convulsions or epilepsy, influenza/ pneumonia, cellulitis and pneumonitis, it is likely that these findings are also relevant to our Western Australian population. A recent report from another Australian state reported that as many as $38 \%$ of deaths in people with intellectual disability were avoidable. ${ }^{41}$

Our findings are similar to others who report the most common causes of death in people with intellectual disability to be circulatory disease, respiratory disease and cancer. ${ }^{15} 41$ They also report higher-than-expected numbers of deaths in these categories compared with the general population and from other ICD chapters 
including diseases of the nervous system. ${ }^{16}$ However, they report no significantly lower results such as we found for cancers and external causes of injuries. This may be due to differing study methodologies.

The similarity of our results to those from other countries as cited above suggests that our results are broadly generalisable internationally. A potential limitation of our study is our ability to accurately identify people with intellectual disability from the IDEA register and administrative health records. However, case ascertainment from the IDEA register has been shown to be good and is consistent with international estimates. ${ }^{18} 19$ Cases identified through the hospital morbidity data system or death certificate alone may have less stringent ascertainment criteria. In future work, it may be useful to distinguish between those with and without severe intellectual disability.

Our research demonstrates that people with intellectual disability continue to experience potentially preventable conditions until the end of their lives and that the health targets presented by Beange et al in 1999 are still relevant today. ${ }^{42}$ The data produced in this study represent a comprehensive method of monitoring progress in preventing disease and complications in people with intellectual disability.

Acknowledgements The authors wish to thank the staff at the Western Australian Data Linkage Branch and the custodians of the Hospital Morbidity Data Collection, the Emergency Department Data Collection, the Mental Health Information System, Intellectual Disability Exploring Answers and the Register of Births, Deaths and Marriages for access to and linkage of their data.

Contributors Study concept and design: LR, HL and JS. Data acquisition and analysis: KB and KS. KB wrote the first draft of the paper, which was then critically reviewed by all authors.

Funding This work was supported by the National Health and Medical Research Council of Australia (grant no. 1084890).

Competing interests None declared.

Patient consent Not required.

Ethics approval Department of Health, Western Australia and Curtin University Human Research Ethics Committee.

Provenance and peer review Not commissioned; externally peer reviewed.

Data sharing statement Access to these data is possible following Human Research Ethics Committee and data custodian approval.

Open Access This is an Open Access article distributed in accordance with the Creative Commons Attribution Non Commercial (CC BY-NC 4.0) license, which permits others to distribute, remix, adapt, build upon this work non-commercially, and license their derivative works on different terms, provided the original work is properly cited and the use is non-commercial. See: http://creativecommons.org/ licenses/by-nc/4.0/

(C) Article author(s) (or their employer(s) unless otherwise stated in the text of the article) 2018. All rights reserved. No commercial use is permitted unless otherwise expressly granted.

\section{REFERENCES}

1. Krahn GL, Hammond L, Turner A. A cascade of disparities: health and health care access for people with intellectual disabilities. Ment Retard Dev Disabil Res Rev 2006;12:70-82.

2. Beange $H$, Bauman $A$. Caring for the developmentally disabled in the community. Can Fam Physician 1990;1555:8-63.

3. Lennox NG, Kerr MP. Primary health care and people with an intellectual disability: the evidence base. J Intellect Disabil Res 1997;41(Pt 5):365-72.
4. lacono T, Bigby C, Unsworth C, et al. A systematic review of hospital experiences of people with intellectual disability. BMC Health Serv Res 2014;14:505.

5. lacono T, Davis R, Humphreys J, et al. GP and support people's concerns and priorities for meeting the health care needs of individuals with developmental disabilities: a metropolitan and nonmetropolitan comparison. J Intellect Dev Disabil 2003;28:353-68.

6. Balogh R, Brownell M, Ouellette-Kuntz $\mathrm{H}$, et al. Hospitalisation rates for ambulatory care sensitive conditions for persons with and without an intellectual disability - a population perspective. J Intellect Disabil Res 2010;54:820-32.

7. Florio T, Trollor J. Mortality among a cohort of persons with an intellectual disability in New South Wales, Australia. J Appl Res Intellect Disabil 2015;28:383-93.

8. Heslop P, Blair PS, Fleming P, et al. The confidential inquiry into premature deaths of people with intellectual disabilities in the UK: a population-based study. Lancet 2014;383:889-95.

9. Tyrer F, McGrother C. Cause-specific mortality and death certificate reporting in adults with moderate to profound intellectual disability. $J$ Intellect Disabil Res 2009;53:898-904.

10. Tyrer F, Smith LK, McGrother CW. Mortality in adults with moderate to profound intellectual disability: a population-based study. $J$ Intellect Disabil Res 2007;51:520-7.

11. Mencap. Death by indifference. London: Mencap, 2007.

12. World Health Assembly. Resolution on disability. Geneva: World Health Organisation, 2013. 66th World Health Assembly, Agenda item 13.5, WHA66.9, 27 May 2013.

13. Council of Australian Governments. National disabilty strategy 2010-2020. Canberra: Council of Australian Governments, 2011.

14. Department of Health. Six Lives. Progress report on healthcare for people with learning disabilities. London: Department of Health, 2013.

15. Glover G, Ayub M. How people with learning disabilities die. Improving Health and Lives: Learning Disabilities Observatory, 2010.

16. Glover G, Williams R, Heslop P, et al. Mortality in people with intellectual disabilities in England. J Intellect Disabil Res 2017;61:62-74.

17. Petterson B, Leonard H, Bourke J, et al. IDEA (Intellectual Disability Exploring Answers): a population-based database for intellectual disability in Western Australia. Ann Hum Biol 2005;32:237-43.

18. Bourke J, de Klerk N, Smith T, et al. Population-based prevalence of intellectual disability and autism spectrum disorders in Western Australia: a comparison with previous estimates. Medicine 2016;95:e3737.

19. Maulik PK, Mascarenhas MN, Mathers CD, et al. Prevalence of intellectual disability: a meta-analysis of population-based studies. Res Dev Disabil 2011;32:419-36.

20. Bourke J, Wong K, Leonard H. Validation of intellectual disability coding through hospital morbidity records using an intellectual disability population-based database in Western Australia. BMJ Open 2018;8:e019113.

21. ICD-10-AM. Tabular list of diseases. Volume 1 of The International Statistical Classification of Diseases and related health problems. 10th Revision, Australian Modification: National Centre for Classification in Health, 2000.

22. Lin E, Balogh R, Cobigo V, et al. Using administrative health data to identify individuals with intellectual and developmental disabilities: a comparison of algorithms. J Intellect Disabil Res 2013;57:462-77.

23. Blackwell M, lacus S, King G, et al. Coarsened exact matching in Stata. Stata Journal 2009;9:524-46.

24. Pink B. Information paper: an introduction to socio-economic indexes for areas (SEIFA) 2006. Canbera: Australia Bureau of Statistics, 2008.

25. Australian Bureau of Statistics. Statistical areas level 1. Statistical geography statistical geography fact sheet. Canberra: Australian Bureau of Statistics, 2012.

26. Glover J, Tennant S. Remote areas statistical geography in Australia: notes on the Accessibility/Remoteness Index for Australia (ARIA+ version). Adelaide: Public Health Information Development Unit, 2003.

27. Elixhauser A, Steiner C, Harris DR, et al. Comorbidity measures for use with administrative data. Med Care 1998;36:8-27.

28. Australian Institute of Health and Welfare. Australian refined diagnosis-related groups (AR-DRG) data cubes. Canberra: Australian Institute of Health and Welfare, 2016.

29. Page A, Ambrose S, Glover J, et al. Atlas of avoidable hospitalisations in Australia: ambulatory care-sensitive conditions: Public Health Information Development Unit, The University of Adelaide, Australian Institute of Health and Welfare, 2007.

30. Purdy S, Griffin T, Salisbury C, et al. Ambulatory care sensitive conditions: terminology and disease coding need to be more specific to aid policy makers and clinicians. Public Health 2009;123:169-73. 
31. Ouellette-Kuntz H, Garcin N, Lewis ME, et al. Addressing health disparities through promoting equity for individuals with intellectual disability. Can J Public Health 2005;96(Suppl 2):S8-22.

32. Beange $\mathrm{H}$, McElduff $A$, Baker W. Medical disorders of adults with mental retardation: a population study. Am J Ment Retard 1995;99:595-604.

33. Balogh RS, Ouellette-Kuntz $\mathrm{H}$, Brownell $\mathrm{M}$, et al. Factors associated with hospitalisations for ambulatory care-sensitive conditions among persons with an intellectual disability: a publicly insured population perspective. J Intellect Disabil Res 2013;57:226-39.

34. Glover G, Evison F. Hospital admissions that should not happen. Admissions for ambulatory care sensitive conditions for people with learning disabilities in England. Improving health and lives: learning disabilities observatory, 2013.

35. Longman JM, Passey ME, Ewald DP, et al. Admissions for chronic ambulatory care sensitive conditions - a useful measure of potentially preventable admission? BMC Health Serv Res $2015 ; 15: 472$
36. Hosking FJ, Carey IM, DeWilde S, et al. Preventable emergency hospital admissions among adults with intellectual disability in England. Ann Fam Med 2017;15:462-70.

37. Sullivan SG, Hussain R, Threlfall T, et al. The incidence of cancer in people with intellectual disabilities. Cancer Causes Control 2004; 15:1021-5.

38. Patja K, Eero P, livanainen M. Cancer incidence among people with intellectual disability. J Intellect Disabil Res 2001;45:300-7.

39. Satgé $\mathrm{D}, \mathrm{Kempf} E$, Dubois JB, et al. Challenges in diagnosis and treatment of lung cancer in people with intellectual disabilities: current state of knowledge. Lung Cancer Int 2016;2016:1-7.

40. Tuffrey-Wijne I, Bernal J, Hubert J, et al. People with learning disabilities who have cancer: an ethnographic study. $\mathrm{Br} J$ Gen Pract 2009;59:503-9

41. Trollor J, Srasuebkul P, Xu H, et al. Cause of death and potentially avoidable deaths in Australian adults with intellectual disability using retrospective linked data. BMJ Open 2017;7:e013489.

42. Beange $\mathrm{H}$, Lennox N, Parmenter TR. Health targets for people with an intellectual disability. J Intellect Dev Disabil 1999;24:283-97. 